\title{
Mortalidad intrahospitalaria en adultos mayores chilenos con fractura de cadera: incidencia, causas y otros elementos de interés
}

\section{Early in-hospital Mortality in Chilean Older People with Hip Fracture}

\author{
José Luis Dinamarca Montecinos ${ }^{1}$ Gedeón Améstica Lazcano ${ }^{2}$ Alejandro Javier Carrasco Buvinic ${ }^{2}$ \\ 1 Programa de Ortogeriatría, Servicio de Ortopedia y Traumatología \\ Adultos, Hospital Dr. Gustavo Fricke, Viña del Mar, Chile \\ 2 Servicio de Ortopedia y Traumatología Adultos, Hospital Dr. Gustavo \\ Fricke, Viña del Mar, Chile \\ Address for correspondence Jose Luis Dinamarca, MD, MSc, PhD, \\ Servicio de Ortopedia y Traumatología Adultos, Hospital Dr. Gustavo \\ Fricke, Álvares 1532, Segundo Piso - Derecha, Viña del Mar, Chile \\ (e-mail: doctordinamarca@yahoo.es).
}

Rev Chil Ortop Traumatol 2018;59:41-46.

\section{Resumen}

\section{Palabras Clave}

- Mortalidad intrahospitalaria

- Fractura de cadera

- Ortogeriatría

\section{Abstract}

Introducción Las fracturas de cadera (FC) presentan alta mortalidad. Existen escasos estudios que profundizan en la mortalidad intrahospitalaria (MIH) asociada.

Objetivo Describir MIH en pacientes $\geq 60$ años con FC.

Materiales y Métodos Corte transversal, descriptivo y analítico, monocéntrico, de colección completa. Fueron evaluados 647 sujetos, de ambos géneros, ingresados con FC entre 01.01.2010 y 31.12.2012, agrupándolos en fallecidos y egresados vivos. Se registró fallecimientos, género, edad, ubicación anatómica, tipo de traumatismo, lugar de ocurrencia de FC, tiempo fractura-fallecimiento, tiempo de estancia hospitalaria, porcentaje de operados, diagnósticos asociados a FC, diagnóstico de fallecimiento, comorbilidad asociada al diagnóstico de fallecimiento y lugar de fallecimiento. Datos en planilla ortogeriátrica, análisis descriptivo.

Resultados $\mathrm{MIH}=3,09 \%$. Edad promedio 84 años. Los diagnósticos de fallecimiento más importantes fueron infecciones (40\%) y enfermedad tromboembólica (15\%). El $80 \%$ presentó comorbilidad crónica relacionada con la causa de fallecimiento. Sin diferencias significativas según edad, género, ubicación anatómica de la FC, lugar de ocurrencia de la FC, tiempo de estancia hospitalaria. Hubo diferencias significativas en cantidad de no operados, mayor en el grupo con $\mathrm{MIH}(p<0.000)$, esperable por sesgo de selección. Es deseable identificar precozmente los sujetos con FC que presentan alto riesgo de MIH. Esto, para definir manejo conservador, optimizar calidad de vida y recursos hospitalarios.

Introduction Hip fractures (HIF), have high rates of early mortality. However, there are few studies that deepen in in-hospital mortality (IHM).

Objective To describe IHM in patients with $\geq 60$ years with HIF. received

January 24, 2018

accepted

July 17, 2018

published online

August 20, 2018
DOI https://doi.org/

10.1055/s-0038-1668593.

ISSN 0716-4548.
Copyright $\odot 2018$ by Thieme Revinter

Publicações Ltda, Rio de Janeiro, Brazil
License terms

(c) (i) $\ominus$ (\$) 


\section{Keywords}

- in-hospital mortality

- hip fracture

- orthogeriatrics
Materials and Methods Cross-sectional, descriptive and analytical, monocentric, full collection. 647 subjects, both genders, admitted with HIF between 01.01.2010 and 12.31.2012, separating them in inhospital deceased and discharged alive. Registered deaths, gender, age, anatomic location, type of trauma, place of occurrence of HIF, time fracture-death, length of hospital stay, percentage of operated, diagnosis associated with HIF, diagnosis of death, comorbidity associated with the diagnosis of death and place of death. Data in orthogeriatric chart, descriptive analysis.

Results $\quad \mathrm{IHM}=3.09 \%$. Average age 84 years. The most important diagnoses of death were infections (40\%) and thromboembolic disease (15\%). $80 \%$ presented chronic comorbidity related to the cause of death. There were no significant differences according to age, gender, HIF anatomical location, place of occurrence of the HIF, length of hospital stay. There were significant differences in the number of nonoperated, higher in the IHM group $(p<0.000)$, expected for selection bias. It is desirable to identify early those subjects with HIF who are at high risk for IHM. This, to define conservative management, optimize quality of life and hospital resources.

\section{Introducción}

Las fracturas de cadera (FC) son un conjunto de enfermedades con incidencia alta y creciente en la población de 60 o más años. ${ }^{1,2}$ En Chile, la incidencia de FC en 2006 fue menor a 150 por cada 100.000 personas de $\geq$ 65 años para ambos géneros, lo que categoriza al país como una zona de bajo riesgo de sufrir $\mathrm{FC}^{3}$ Sin embargo, en la región estudiada, entre 2010-2012 la incidencia fue 177/ 100.000 personas de $\geq 65$ años para ambos géneros, calificando como de riesgo moderado. ${ }^{4}$

Además de alta morbilidad asociada, presentan alta mortalidad, que a 1 año varía entre $18 \%$ y $30 \%^{5-8} \operatorname{Sin}$ embargo, los estudios sobre mortalidad intrahospitalaria (MIH) asociada a FC son escasos, generalmente retrospectivos $\mathrm{y}$, habitualmente, tratan a la MIH como una variable más dentro del contexto de caracterizaciones epidemiológicas generales de muestras de pacientes con FC, refiriéndose a ella con relación a su incidencia. ${ }^{9-11}$

Por diversos motivos, MIH por FC es analizada como parte de la llamada mortalidad temprana a 30 días, ${ }^{12}$ aunque en diversos países es considerada desde hace tiempo indicador de calidad del manejo hospitalario, ${ }^{13,14}$ Como la estancia hospitalaria de los pacientes con FC en Chile es alta, ${ }^{4}$ formando parte importante de los primeros 30 días posteriores a la fractura, MIH debiera considerarse como indicador de la calidad del manejo general de las FC. Entre otras razones, pues es verificable, confiable, sencilla de medir y útil: Generalmente, puede limitarse conociendo las variables que la determinan e implementando acciones para minimizar o anular su efecto. Dichas medidas benefician la calidad de vida de pacientes y familiares; facilitan decisiones del equipo de salud y optimizan recursos hospitalarios.

Por ello es importante conocer los factores locales asociados a la MIH. Al no encontrar estudios que caractericen mortalidad intrahospitalaria $(\mathrm{MIH})$ de las $\mathrm{FC}$ en población chilena, decidimos estudiarla, describiendo las características generales de pacientes mayores con FC que fallecieron durante su estancia hospitalaria; evaluando si existen diferencias significativas entre los pacientes fallecidos y los que egresaron vivos en el mismo periodo, y contrastando resultados con la bibliografía disponible.

\section{Material y Métodos}

Diseño: Transversal. Observacional, descriptivo y analítico. Monocéntrico.

Se estudió el trienio 01.01.2010-31.12.2012. Serie de 647 sujetos chilenos, $\geq 60$ años, ambos géneros, que ingresaron en forma consecutiva al Hospital con diagnóstico imagenológico de FC realizado por traumatólogo. Los datos se obtuvieron conforme los pacientes ingresaban al Hospital, tras firmar consentimiento informado. Se contrastó el listado obtenido con registro del sistema estadístico del Hospital para códigos CIE-10 S72.0 (fractura de cuello de fémur), S72.1 (pertrocanteriana) y S72.2 (subtrocanteriana).

De esa muestra, se seleccionó una submuestra compuesta por los fallecidos durante la hospitalización.

\section{Variables}

1. Género

2. Edad

3. Días de estancia

4. Días entre FC y fallecimiento.

5. Ubicación anatómica de FC (extracapsulares/ intracapsulares)

6. Tipo de traumatismo asociado (mínimo, de alta energía, no traumática).

7. Lugar de ocurrencia de FC (domicilio, vía pública, trabajo, otro)

8. Diagnósticos etiológicos de FC:

a. Osteoporosis primaria,

b. Osteoporosis secundaria: albúmina 2,5 g\% o menos, uso crónico de corticoides o antiepilépticos

c. Osteodistrofia renal 

d. Grandes traumatismos
e. Neoplasias

9. Paciente operado o no operado

10. Causa de no operar

11. Diagnóstico de fallecimiento (consignado en Certificado de Defunción o Ficha Clínica por el médico que constató el fallecimiento).

12. Comorbilidad, consignada en Ficha, relacionada con diagnóstico de fallecimiento.

13. Lugar de fallecimiento en el hospital.

Las FC se consideraron osteoporóticas en ausencia de osteodistrofia renal y fractura neoplásica. Los sujetos con diagnóstico densitométrico previo de osteoporosis se incluyeron en ese grupo. Se consideró osteoporosis secundaria si los pacientes tenían factores de riesgo (fármacos, hipoalbuminemia); y primaria si no.

Al no contar con densitómetro, el diagnóstico de osteoporosis se realizó con los criterios de pesquisa clínica del National Osteoporosis Guideline Group (NOGG): Las fracturas mayores por fragilidad en mujeres postmenopáusicas y varones $\geq 50$ años, en ausencia de densitometría ósea, deben considerarse osteoporóticas. ${ }^{15}$

\section{Manejo de Los Datos}

Traspasados a planilla Excel (versión 15). Estadística descriptiva y analítica. El análisis se hizo con SPSS (versión
22), realizándose comparación en función de proporciones con Chi cuadrado $(p<0,05)$ y valores medios a través de prueba $\mathrm{t}$, buscando diferencias entre fallecidos y egresados vivos.

\section{Resultados}

\section{Descriptivos}

\section{Muestra}

Se analizaron 647 sujetos, 76,2\% mujeres, edad promedio 80,8 años, que utilizaron 10,898 días cama. Y 113 sujetos no operados (17,5\%). Otras frecuencias, en - Tablas 1 y 2.

\section{Submuestra}

MIH: $3.09 \%(n=20)$.

Un 80\% mujeres. Edad promedio 83,75 años (66-100 años, DT $=8,49)$; varones 84,5 años, mujeres 83,6 años. Ocuparon 260 días cama (media $=13,2,4 \%$ de la muestra).

Entre FC y fallecimiento transcurrieron en promedio 19,2 días.

Tipo de FC: 65\% extracapsular, 35\% intracapsular.

Todos los fallecidos tuvieron como origen de la FC un traumatismo de baja intensidad.

Un $90 \%$ de FC domiciliarias. Restantes ocurrieron en la calle.

Diagnósticos etiológicos asociados a FC: Osteoporosis primaria $60 \%(n=12)$; secundaria $20 \%$ (hipoalbuminemia

Tabla 1 Analítica comparativa entre sujetos fallecidos y egresados vivos en función de proporciones en mayores chilenos hospitalizados con Fractura de Cadera (Viña del Mar, Chile 2010-2012)

\begin{tabular}{|l|l|l|l|l|l|l|l|}
\hline Variable & Fallecidos & $\begin{array}{l}\text { Egresados } \\
\text { vivos }(\%)\end{array}$ & Chi $^{2}$ & gl & p valor & \multicolumn{2}{l|}{ IC al 95\% } \\
\cline { 4 - 8 } & & & & & Límite superior & Límite inferior \\
\hline Sexo (mujer) & 81.0 & 76.0 & 0.2705 & 1 & 0.6030 & -0.1221 & 0.2203 \\
\hline Ubicación anatómica (FEC) & 66.7 & 66.3 & 0.0013 & 1 & 0.9716 & -0.2012 & 0.2087 \\
\hline No operados & 81.0 & 15.3 & 60.6890 & 1 & 0.0000 & 0.4858 & 0.8264 \\
\hline Lugar de ocurrencia (domicilio) & 90.5 & 91.9 & 0.0512 & 1 & 0.8210 & -0.1411 & 0.1135 \\
\hline $\begin{array}{l}\text { Principal tipo de traumatismo } \\
\text { (mínima energí) }\end{array}$ & 100.0 & 91.4 & 1.9765 & 1 & 0.1598 & 0.0642 & 0.1082 \\
\hline Osteoporosis primaria & 61.9 & 65.0 & 0.0864 & 1 & 0.7689 & -0.2421 & 0.1799 \\
\hline Osteodistrofia renal & 9.5 & 2.9 & 2.9980 & 1 & 0.0833 & -0.0597 & 0.1927 \\
\hline DSP & 9.5 & 10.9 & 0.3777 & 1 & 0.8460 & -0.1412 & 0.1145 \\
\hline Fármacos o sustancias & 9.5 & 12.0 & 0.1170 & 1 & 0.7323 & -0.1526 & 0.1035 \\
\hline Grandes traumatismos & 0.0 & 3.8 & 0.8361 & 1 & 0.3605 & 0.0232 & 0.0633 \\
\hline Neoplasias & 0.0 & 2.4 & 0.5151 & 1 & 0.4729 & -0.0359 & -0.0119 \\
\hline Hiperparatiroidismo primario & 0.0 & 0.6 & 0.1350 & 1 & 0.7133 & -0.0126 & 0.0001 \\
\hline
\end{tabular}

Tabla 2 Analítica comparativa entre sujetos fallecidos y egresados vivos con valores medios en mayores chilenos hospitalizados con Fractura de Cadera (Viña del Mar, Chile 2010-2012)

\begin{tabular}{|l|l|l|l|l|l|l|l|}
\hline Variable (\%) & MIH & Egresados vivos & $\mathbf{t}$ & gl & p valor & \multicolumn{2}{|l|}{ IC al 95\% } \\
\cline { 5 - 9 } & & & & & & Límite superior & Límite inferior \\
\hline Edad & 84 & 80.7 & 1.742 & 21.25 & 0.0959 & -06280 & 7.2500 \\
\hline Días de estancia en el Hospital & 13.52 & 17 & -2.065 & 23.34 & 0.0501 & -6.9615 & 0.0027 \\
\hline
\end{tabular}


severa $10 \%$; fármacos $10 \%)$; osteodistrofia renal $10 \%(n=2)$; otros $10 \%(n=2)$.

En $80 \%$ de los fallecidos $(n=16)$ se había decidido no operar. En $15 \%(n=3)$ se realizó cirugía, $5 \%(n=1)$ falleció previo a cirugía programada.

Los 16 sujetos no operados que presentaron $\mathrm{MIH}$ representan el $14,16 \%$ del total de sujetos no operados.

Las causas de no operar fueron: $20 \%$ alto riesgo de mortalidad a 30 días (medido con Nottingham Hip Fracture Score), 30\% mínimo impacto funcional (estimado con Índice de Barthel previo), 50\% ambas causas.

Un $90 \%$ con causa de muerte establecida: Infecciones del $45 \%$ (35\% neumonía intrahospitalaria, 10\% pielonefritis aguda); tromboembolismo pulmonar (TEP) 15\%; hiperkalemia severa, infarto agudo al miocardio (IAM) y hemorragia digestiva alta (HDA) cada uno $10 \%$.

Un $80 \%$ presentó comorbilidad relacionada con el diagnóstico de muerte, descrita como antecedente al ingreso.

Los fallecimientos ocurrieron en la sala de Traumatología (80\%), Unidad de Paciente Crítico (UPC) (10\%), 5\% pabellón previo a cirugía programada, $5 \%$ otro servicio (Medicina Interna).

\section{Analíticos}

Sobre la comparación en función de proporciones (porcentajes) la -Tabla $\mathbf{1}$ dispone las comparaciones realizadas. La única diferencia significativa se da entre proporción de no operados.

Sobre el cálculo de diferencias significativas usando valores medios, los resultados se disponen en - Tabla 2, no existiendo diferencias significativas entre fallecidos y egresados vivos en cuanto a edad ni días de estancia hospitalaria.

\section{Discusión y Comentarios}

La muestra es pequeña, acotando potencia del estudio. La única diferencia entre fallecidos y no fallecidos fue el número de no operados. Sin embargo, al ser el riesgo vital elevado un criterio para decidir el manejo no quirúrgico, esa diferencia debe interpretarse como artificial y esperable: Los sujetos no operados fallecieron significativamente más porque tenían mayor riesgo de mortalidad, razón por la cual fueron seleccionados para manejo no quirúrgico.

Es destacable que el porcentaje de no operados que falleció en el hospital representa un porcentaje menor del total de sujetos no operados, lo que probablemente se debe a sistemas adecuados de selección de sujetos para manejo no quirúrgico. Aunque no fue motivo del presente trabajo, teniendo en cuenta el alto riesgo general de esos sujetos, con una mortalidad anual que llega hasta $30 \%$, es dable comentar que es fundamental contar con instrumentos que apoyen esta decisión. Al respecto, la literatura enfatiza la utilidad de los programas de Ortogeriatría en la disminución de la MIH y selección de pacientes. ${ }^{16-18}$

Otros resultados se comentan según contraste con la literatura.

La incidencia de $\mathrm{MIH}$ se asemeja a publicaciones estadounidenses, europeas y japonesas. ${ }^{9-11,19-22}$ La mortalidad temprana a 30 días reportada varía entre 2,5 y
13,3\%, con promedios de $8 \% .^{12,23}$ Considerando otras publicaciones, y que MIH es una fracción de esta mortalidad a 30 días, parece razonable que permanezca bajo $5 \%{ }^{24} \operatorname{Sin}$ embargo, como indicador, $\mathrm{MIH}$ representa una utilidad particular con relación a las medidas a implementar en los centros hospitalarios. ${ }^{13,14}$

Se ha identificado cirugía $\operatorname{precoz}^{10}$ y manejo ortogeriátrico $^{16-18}$ como responsables de la disminución de MIH en FC. La baja MIH de esa muestra puede estar relacionada con la existencia de un programa de Ortogeriatría desde 2009 en el centro estudiado. Pero no se cuenta con datos de $\mathrm{MIH}$ previa que confirmen esta afirmación.

Serra y col., ${ }^{19}$ describen doble MIH para varones con FC. No todos los trabajos que buscan determinar riesgo de mortalidad en FC toman en cuenta el género, que es un elemento controvertido, ${ }^{20,25,26}$ no quedando claro si ser varón es o no factor de riesgo.

La edad desde 80 años (por el índice SERNBO), ${ }^{27}$ u 86 (por índice de Nothingham ${ }^{28}$ y score de Almelo) ${ }^{29}$ sí es considerada un elemento contundente. En esta muestra, MIH aumenta desde 80 años en ambos sexos. Pero si tomamos como corte la edad sugerida por Nothingham, la muestra se encuentra en promedio bajo ella. Aunque eso podría colaborar con la baja incidencia de MIH encontrada, debe considerarse que SERNBO fue construido para medir riesgo de mortalidad temprana solo en fracturas intracapsulares. Por otra parte, el índice de Nottingham se utiliza para medir riesgo de mortalidad a 30 días tras la reparación quirúrgica de la $\mathrm{FC}^{30}$

La ausencia de diferencia estadística en cuanto lugar de ocurrencia de FC y ubicación anatómica es consistente con la literatura. ${ }^{19,31-33}$

El porcentaje de sujetos con FC que no se opera es variable, entre $11 \%-34 \% .{ }^{34}$ En la presente serie, en un alto porcentaje de los pacientes fallecidos se había decidido previamente no operar. Eso se realizó en conjunto con Geriatría y familiares del paciente fracturado, teniendo en cuenta fundamentalmente parámetros de rendimiento funcional post quirúrgico, calidad de vida y riesgo vital a 30 días.

Foss y col., ${ }^{33}$ describen que hasta el $43 \%$ de esos fallecimientos precoces puede ser considerado inevitable debido al mal estado general de los pacientes con FC. La decisión de no operar permite un mejor uso de recursos una vez seleccionados esos pacientes. Por ello, habiéndose decidido manejo no quirúrgico, el traslado a un servicio médico puede mejorar la calidad de vida de esos pacientes; lo mismo el traslado a domicilio en casos seleccionados. ${ }^{35}$ En esta serie no se otorgó altas domiciliarias y solo $15 \%$ de los sujetos fue trasladado a otro Servicio del Hospital. Eso es un aspecto mejorable, toda vez que uno de los temores más frecuentemente identificados por la población mayor es no fallecer en su propio domicilio o fallecer en un hospital. ${ }^{36}$

Las complicaciones más frecuentemente reportadas en muestras generales de FC fueron infecciones urinarias $\mathrm{y}$ respiratorias, delirium, descompensaciones cardiorespiratorias, enfermedad tromboembólica $\mathrm{y}$ hemorragia digestiva, en ese orden. ${ }^{31}$ Todas ellas (salvo delirium) fueron identificadas como causales de $\mathrm{MIH}$ en esta muestra. Así, las 
enfermedades responsables de MIH no serían rarezas sino entidades conocidas y de incidencia frecuente y esperable en pacientes con FC. Es destacable la aparición de la hemorragia digestiva como causa de $\mathrm{MIH}$, pues estos pacientes reciben anticoagulación.

Aunque no se encontraron publicaciones que dieran cuenta de los diagnósticos asociados a MIH de FC, sí hay reportes de mortalidad a 30 días: Distintas revisiones ${ }^{37,38}$ describen que las causas más frecuentes son neumonías y trastornos cardiovasculares como infarto miocárdico e insuficiencia cardíaca; destacando como menos frecuentes sepsis, hemorragia digestiva y enfermedad tromboembólica. Mismas causas encontradas en la presente serie.

Clínicamente es un aspecto positivo contar con una $\mathrm{MIH}$ baja, pero eso supone la principal dificultad del presente estudio: A pesar de ser un trienio el período revisado, hemos obtenido una muestra pequeña. No obstante, es interesante constatar la similitud de esos resultados con lo descrito en trabajos con muestras mayores, y constatar la acción ortogeriátrica con relación a las decisiones de no operar.

Por otra parte, aunque MIH es solo una fracción de la mortalidad general, y muchos pacientes con FC fallecen (operados y no operados) fuera del hospital tanto en el corto como el mediano plazo, MIH debe ser vista como un indicador sobre el que actuar para mejorar parámetros tanto de calidad de vida como de equidad en la distribución de los recursos hospitalarios. Posiblemente no sea evitable la muerte, ${ }^{33}$ pero sí es posible evitar que el paciente fallezca en el hospital, y entregar una mejor calidad de vida al paciente y a su familia. En ese sentido se hacen indispensables los programas de asistencia domiciliaria.

Como comentarios finales, podemos referir que esta muestra presentó baja MIH. Los pacientes que fallecieron tenían entre 80-90 años y comorbilidad crónica relacionada con el fallecimiento. Las principales causas de MIH fueron condiciones frecuentes y esperables en ese tipo de pacientes, como infecciones respiratorias y enfermedad tromboembólica.

$\mathrm{MIH}$ debe considerarse un indicador de calidad en el manejo agudo de FC en personas mayores. Es de interés identificar a los sujetos con mayor riesgo de sufrir $\mathrm{MIH}$, pues es un argumento para definir si procederá o no el manejo quirúrgico. Calidad de vida, costes y recursos pueden optimizarse con una adecuada definición, aunque esta debe ser lo más precoz posible. El manejo ortogeriátrico y la utilización de instrumentos específicos de valoración de riesgo vital en FC son herramientas valiosas.

\section{Financiación}

Los autores declaran ausencia de financiamientos para esta investigación.

\section{Conflictos de interés}

Ninguno.

\section{Bibliografía}

1 Porto-Carriero F, Christmas C. In the Clinic: Hip Fracture. Ann Intern Med 2011;155(11):ITC6-1-ITC6-15
2 Cornwall R, Gilbert MS, Koval KJ, Strauss E, Siu AL. Functional outcomes and mortality vary among different types of hip fractures: a function of patient characteristics. Clin Orthop Relat Res 2004;(425):64-71

3 Kanis JA, Odén A, McCloskey EV, Johansson H, Wahl DA, Cooper C; IOF Working Group on Epidemiology and Quality of Life. A systematic review of hip fracture incidence and probability of fracture worldwide. Osteoporos Int 2012;23(09):2239-2256

4 Dinamarca-Montecinos JL, Améstica-Lazcano G, Rubio-Herrera R, Carrasco-Buvinic A, Vásquez A. Hip fracture. Experience in 647 Chilean patients aged 60 years or more. Rev Med Chil 2015;143 (12):1552-1559

5 Brozek W, Reichardt B, Kimberger O, et al. Mortality after hip fracture in Austria 2008-2011. Calcif Tissue Int 2014;95(03):257-266

6 Bredahl C, Nyholm B, Hindsholm KB, Mortensen JS, Olesen AS. Mortality after hip fracture: results of operation within $12 \mathrm{~h}$ of admission. Injury 1992;23(02):83-86

7 Cree M, Soskolne CL, Belseck E, et al. Mortality and institutionalization following hip fracture. J Am Geriatr Soc 2000;48(03):283-288

8 Hamlet WP, Lieberman JR, Freedman EL, Dorey FJ, Fletcher A, Johnson EE. Influence of health status and the timing of surgery on mortality in hip fracture patients. Am J Orthop 1997;26(09):621-627

9 Belmont PJJr, Garcia EJ, Romano D, Bader JO, Nelson KJ, Schoenfeld AJ. Risk factors for complications and in-hospital mortality following hip fractures: a study using the National Trauma Data Bank. Arch Orthop Trauma Surg 2014;134(05):597-604

10 O'Connor I, McDowell D, Barnes D. Hip Fractures: The St Ann's Bay Regional Hospital Experience. West Indian Med J 2014;63(02): 138-141

11 Martínez-Rondanelli A. Fracturas de cadera en ancianos: pronóstico, epidemiología. Aspectos generales: experiencia. Rev Col de Or Tra 2005;19(01):20-28. Disponible en: http://www. sccot.org.co/pdf/RevistaDigital/19-01-2005/05Fracturas.pdf

12 Giannoulis D, Calori GM, Giannoudis PV. Thirty-day mortality after hip fractures: has anything changed? Eur J Orthop Surg Traumatol 2016;26(04):365-370

13 Guide to Inpatient Quality Indicators: Quality of Care in Hospitals Volume, Mortality, and Utilization. Department of Health and Human Services. Agency for Healthcare Research and Quality, June 2002. Version 3.1 (March 12, 2007), pags 56-57. http:// www.qualityindicators.ahrq.gov

14 Sociedad Española de Calidad Asistencial. Indicadores de Calidad para Hospitales del Sistema Nacional de Salud, marzo 2012, p 65. Disponible en: http://www.calidadasistencial.es/images/gestion/ biblioteca/335.pdf

15 Compston J, Cooper A, Cooper C, et al. Guideline for the diagnosis and management of osteoporosis. The National Osteoporosis Guideline Group, 2014

16 Zeltzer J, Mitchell RJ, Toson B, Harris IA, Ahmad L, Close J. Orthogeriatric services associated with lower 30-day mortality for older patients who undergo surgery for hip fracture. Med J Aust 2014;201(07):409-411

17 Vidán MT, Vaquero J. Unidades de ortogeriatría: Impacto en los resultados de la fractura de cadera en el anciano. En Fracturas femorales en el anciano. Cap 4, 51-56. 2013 SECOT-AAOS. Disponible en http://www.secot.es/descargas/monografias/ 2013/monografia_2013_3.pdf

18 Tarrant SM, Hardy BM, Byth PL, Brown TL, Attia J, Balogh ZJ. Preventable mortality in geriatric hip fracture inpatients. Bone Joint J 2014;96-B(09):1178-1184

19 Serra JA, Garrido G, Vidán M, Marañón E, Brañas F, Ortiz J. Epidemiology of hip fractures in the elderly in Spain. An Med Interna 2002;19(08):389-395

20 Pioli G, Barone A, Giusti A, et al. Predictors of mortality after hip fracture: results from 1-year follow-up. Aging Clin Exp Res 2006; 18(05):381-387 
21 Shoda N, Yasunaga H, Horiguchi H, et al. Risk factors affecting inhospital mortality after hip fracture: retrospective analysis using the Japanese Diagnosis Procedure Combination Database. BMJ Open 2012;2(03):e000416

22 Sancho CA, Arguedas C. Epidemiología de la fractura de cadera de origen osteoporótico en Costa Rica en un período de cinco años. Rev Esp Enferm Metab Oseas 2000;2(09):66-69. Disponible en: http://www.elsevier.es/en-revista-reemo-70-articuloepidemiologia-de-la-fractura-de-10016982

23 Hu F, Jiang C, Shen J, Tang P, Wang Y. Preoperative predictors for mortality following hip fracture surgery: a systematic review and meta-analysis. Injury 2012;43(06):676-685

24 Myers AH, Robinson EG, Van Natta ML, Michelson JD, Collins K, Baker SP. Hip fractures among the elderly: factors associated with in-hospital mortality. Am J Epidemiol 1991;134(10):1128-1137

25 Choi HG, Lee YB, Rhyu SH, Kwon BC, Lee JK. Mortality and cause of death postoperatively in patients with a hip fracture. Bone Joint J 2018 Apr 1;100-B(04):436-442

26 Aharonoff GB, Koval KJ, Skovron ML, Zuckerman JD. Hip fractures in the elderly: predictors of one year mortality. J Orthop Trauma 1997;11(03):162-165

27 Dawe EJ, Lindisfarne E, Singh T, McFadyen I, Stott P. Sernbo score predicts survival after intracapsular hip fracture in the elderly. Ann R Coll Surg Engl 2013;95(01):29-33

28 Moppett IK, Wiles MD, Moran CG, Sahota O. The Nottingham Hip Fracture Score as a predictor of early discharge following fractured neck of femur. Age Ageing 2012;41(03):322-326

29 Nijmeijer WS, Folbert EC, Vermeer M, Slaets JP, Hegeman JH. Prediction of early mortality following hip fracture surgery in frail elderly: The Almelo Hip Fracture Score (AHFS). Injury 2016;47 (10):2138-2143
30 Moppett IK, Parker M, Griffiths R, Bowers T, White SM, Moran CG. Nottingham Hip Fracture Score: longitudinal and multiassessment. Br J Anaesth 2012;109(04):546-550

31 Kesmezacar H, Ayhan E, Unlu MC, Seker A, Karaca S. Predictors of mortality in elderly patients with an intertrochanteric or a femoral neck fracture. J Trauma 2010;68(01):153-158

32 Karagiannis A, Papakitsou E, Dretakis K, et al. Mortality rates of patients with a hip fracture in a southwestern district of Greece: ten-year follow-up with reference to the type of fracture. Calcif Tissue Int 2006;78(02):72-77

33 Altadill Arregui A, Gomez Alonso C, Virgós Soriano MJ, Diaz López B, Cannata Andía JB. Epidemiology of hip fracture in Asturias. Med Clin (Barc) 1995;105(08):281-286

34 Foss NB, Kehlet $\mathrm{H}$. Mortality analysis in hip fracture patients: implications for design of future outcome trials. $\mathrm{Br} \mathrm{J}$ Anaesth 2005;94(01):24-29

35 Kondo A, Yamaguchi C, Fujimoto E. Relationship between admission day and timing of surgery for patients with hip fracture. Jpn J Nurs Sci 2014;11(04):248-258

36 Ceballos García G, Castro Campillo G. La muerte en el anciano. Cap 9, “el lugar de la muerte", pp 67-75. Tesina de Diplomatura en Tanatología. Ediciones de la Asociación Mexicana de Tanatología y Asociación Mexicana de educación continua y a distancia. México DF, 2014. Disponible en http://www.tanatologia-amtac.com/desc argas/tesinas/149\%20La\%20muerte.pdf

37 González-Montalvo JI, Alarcón T, Hormigo Sánchez AI. Why do hip fracture patients die? Med Clin (Barc) 2011;137(08): 355-360

38 Chatterton BD, Moores TS, Ahmad S, Cattell A, Roberts PJ. Cause of death and factors associated with early in-hospital mortality after hip fracture. Bone Joint J 2015;97-B(02):246-251 